\title{
Sensitivity Enhancement of Silver Based Surface Plasmon Resonance Sensor via Graphene-Dielectric Composite Structure
}

\author{
Guiqiang Wang ${ }^{1,2, *}$, Liang Huang ${ }^{1,2}$ \\ ${ }^{1}$ School of Instrument Science and Optoelectronics Engineering, Hefei University of Technology, Hefei \\ 230009, Anhui, China \\ ${ }^{2}$ Anhui Province Key Laboratory of Measuring Theory and Precision Instrument, Hefei University of \\ Technology, Hefei 230009, Anhui, China \\ *corresponding author. Email: wgqiang@hfut.edu.cn
}

\begin{abstract}
In this paper, a silver based surface plasmon resonance (SPR) sensor with graphene and dielectric layer was presented. The influences of dielectric layer and graphene on sensitivity and other sensing properties were theoretically calculated and then comprehensively discussed. The refractive index sensitivities for composite silver film based SPR sensors with graphene and dielectric layer could be increased by $29 \%$ and $288 \%$ than that of monolayer silver film based SPR sensor, respectively. Further, the sensitivity could be enhanced by $202 \%$ when combining graphene and dielectric layer together. Considering the high adsorptive capacity of graphene for biochemical molecules, the composite silver film with both dielectric layer and graphene would have great potential application in biochemical sensing fields. Further, BSA protein was successfully used to verify the biochemical sensing ability of proposed SPR sensor. The shift of resonance angle is nearly 3.1 folds than that of monolayer silver based SPR sensor.
\end{abstract}

Keywords Surface plasmon resonance sensor; Graphene; Dielectric layer; Sensitivity; BSA detection 


\section{Introduction}

Silver based surface plasmon resonance (SPR) sensor has been widely studied since its more excellent sensing performance than that of traditional gold based SPR sensor [1, 2]. However, its poor chemical stability limits the further application. In order to protect silver film in stability, these structures of silver/gold double layer [3-5], silver/metal oxide layer [6, 7], silver/organic molecular layer [8,9] and silver/other dielectric layer [10-12] have been employed. In order to ensure the stability of the silver film, a thick dielectric layer is usually required, which will limit the optimization of sensing performance in terms of thickness.

Two-dimensional nanomaterials have also been widely used in the field of SPR sensor due to their excellent optical and electrical properties, such as optical band transparency and high specific surface area [13-15]. The composite structure based on silver and two-dimensional nanomaterials has also attracted much attention [16, 17]. The typical silver/graphene structure and silver/TMDCs (transitional metal dichalcogenides) structure have not only improved the refractive index (RI) sensitivity of the silver based SPR sensor, but also could improve its stability. Wu et al. theoretically calculated the RI sensitivity of the silver/BP (black phosphorus)/graphene/TMDCs based SPR biochemical sensor, and the introduction of two-dimensional nanomaterials such as BP, graphene or TMDCs improved the RI sensitivity with more than $200 \mathrm{deg} / \mathrm{RIU}($ refractive index unit) [18]. Further, Xia et al. used genetic algorithm to optimize the sensing structure, and when the thicknesses of silver film and BP are $49 \mathrm{~nm}$ and 12 layers (approximately $6 \mathrm{~nm}$ ), the RI sensitivity could reach as high as $400 \mathrm{deg} / \mathrm{RIU}$ [19]. Wu et al. also improved the stability and sensitivity of the silver based SPR sensor via introducing several layers of twodimensional $\mathrm{Ti}_{3} \mathrm{C}_{2} \mathrm{~T}_{\mathrm{x}}$ MXenes, and theoretical calculations showed that the RI sensitivity was $28.4 \%$ higher than that of the conventional silver based SPR sensor [20].

Graphene is a special two-dimensional nanomaterial, due to its high specific surface area, a theoretical value of $2630 \mathrm{~m}^{2} / \mathrm{g}$, which is conducive to increasing the adsorption capacity of biomolecule [21-23]. Szunerits et al. successfully modified the 
graphene surface with biotinylated bovine serum protein (BSA), and the adsorption capacity is 5 folds higher than that of gold membrane [21]. Owing to its high density and excellent optical properties, the introduction of graphene would provide the possibility of realizing high stability and high sensitivity for silver film based SPR sensing $[4,24,25]$.

In summary, the introduction of dielectric layer and two-dimensional nanomaterials can improve the stability and sensitivity of the silver based SPR sensor [26]. However, few research investigates their effects on sensing performance when considering both dielectric layer and two-dimensional nanomaterials (it is worth noting that the dielectric has not an imaginary part of RI and the RI of the two-dimensional nanomaterials is complex in the paper). Further, since a certain thickness of dielectric layer is required to protect silver film better and the defects in two-dimensional nanomaterials reduces the actual protection effect, we presented a structure of graphene/dielectric/silver/BK7 based silver SPR sensor to enhance stability. The introduction of graphene can protect both the silver film and dielectric layer, and make it possible to optimize the sensing structure of silver based SPR sensor.

\section{Theoretical Modeling of the Proposed Sensor}

In this proposed composite silver film based SPR sensor, the Kretschmann configuration via BK7 prism [9] is used to excite SPR mode, as shown in Fig. 1. The transfer matrix method is employed to calculate its reflectivity versus incident angle spectrum based on Fresnel reflection theory [27]. And then, its sensing performance would be calculated based on these angular spectra. In order to enhance the stability of silver film, a 2-nm Cr layer is deposited on the BK7 glass as an adhesion layer before the deposition of silver film [9]. Thus, the Cr film is added in our sensing structure. The wavelength of incident light with transverse magnetic (TM) mode is set as $633 \mathrm{~nm}$, and thus the corresponding calculation parameters of different layers, including refractive index and thickness, are shown in Table 1. The thickness of graphene monolayer is 0.34 nm. 


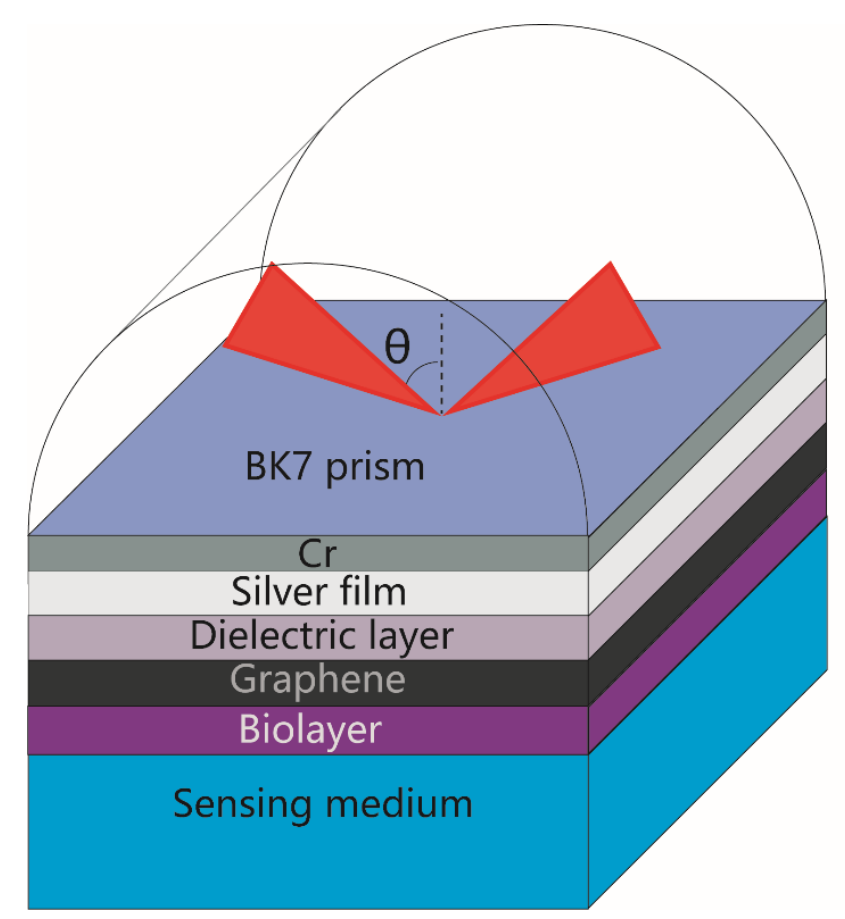

Fig. 1 Schematic diagram of proposed silver based SPR sensor with graphene/dielectric/silver/BK7 structure

Table 1 The parameters of different layers for calculation

\begin{tabular}{ccc}
\hline Layer & Refractive index & Thickness (nm) \\
\hline BK7 glass & $1.515[28]$ & $\infty$ \\
Cr & $3.1395+\mathrm{i} 3.3152[29]$ & 2 \\
Silver film & $0.056206+\mathrm{i} 4.2776[30]$ & $10 \sim 90$ \\
Dielectric layer & $1.35 \sim 5$ & $0 \sim 30$ \\
Graphene & $3.0+\mathrm{i} 1.1491[31]$ & $0 \sim 12$ layers \\
Sensing medium (water) & $1.33+\triangle \mathrm{n}$ & $\infty$ \\
\hline
\end{tabular}

The evaluation indexes for sensing performance we used are RI sensitivity, figure of merit (FoM), penetration depth, field enhancement and RI range. The RI sensitivity is given as [32]:

$$
\mathrm{S}=\frac{\Delta \theta_{\mathrm{R}}}{\Delta \mathrm{n}}
$$

where $\mathrm{S}$ represents the RI sensitivity, $\Delta \theta_{R}$ is the difference of resonance angle with the RI change of sensing medium, that is $\Delta n_{s}=1.335-1.33=0.005$ RIU. Based on 
the obtained RI sensitivity, the FoM is [33]:

$$
\mathrm{FoM}=\frac{\mathrm{S}}{2 \times \text { LHWHM }}
$$

where LHWHM is the left half width at half maximum (LHWHM). Since the SPR angular spectrum is asymmetric and the LHWHM is easier to be obtained than that of the right half width at half maximum, the LHWHM is adopted rather than full width at half maximum. The electric field intensity decays in the sensing medium and the penetration depth is defined as the distance at where the electric field intensity is 1/e of that at the sensor/sensing medium interface. The electric field intensity enhancement factor $(E F)$ in this paper is the averaged electric field intensity enhancement within 20 $\mathrm{nm}$ from the sensor/sensing medium interface into the sensing medium, that is:

$$
\mathrm{EF}=\frac{\int|E|^{2} \mathrm{dz}}{\left|E_{0}\right|^{2} \int d z}
$$

where $\mathrm{E}$ is electric field intensity, $\mathrm{z}$ is normal distance and $\mathrm{E}_{0}$ is electric field intensity at the BK7/Cr interface. For simplicity, the field enhancement would be used instead of EF in the following paragraphs.

The BSA protein was used for sensing application of the optimized silver based SPR sensor. Assuming homogeneous adsorption of BSA molecule on the sensor's outer surface, the RI of the closely packed BSA monolayer could be calculated to be $\mathrm{n}_{\mathrm{BSA}}=$ $1.44+5000 / \lambda^{2}$ and $\lambda$ is the wavelength of incident beam [34]. It was calculated to be 1.452 when the wavelength of incident light is $633 \mathrm{~nm}$. The RI of the BSA adlayer $\left(\mathrm{n}_{\mathrm{ad}}\right)$ was calculated by using the Lorentz-Lorenz equation described below [34, 35]:

$$
\frac{\mathrm{n}_{\mathrm{ad}}^{2}-1}{\mathrm{n}_{\mathrm{ad}}^{2}+2}=\frac{\mathrm{n}_{\mathrm{BSA}}^{2}-1}{\mathrm{n}_{\mathrm{BSA}}^{2}+2} \mathrm{f}_{\mathrm{BSA}}+\frac{\mathrm{n}_{\mathrm{s}}^{2}-1}{\mathrm{n}_{\mathrm{s}}^{2}+2}\left(1-\mathrm{f}_{\mathrm{BSA}}\right)
$$

where $f_{B S A}$ presents the volume fraction of adsorbed BSA in the monolayer adlayer.

\section{Sensing Effects of Multilayer Structure with Dielectric and Graphene}

Before optimizing the graphene/dielectric/silver/BK7 based SPR sensor, the structure of graphene/silver/BK7 and dielectric/silver/BK7 were calculated for comparison. 
For calculating the structure of graphene/silver/BK7, the number of graphene layers is set as $0 \sim 12$ layers and other parameters are shown in Table 1. The results in Fig. 2 indicate that the thickness of silver film and the layers of graphene have significant influences on the RI sensitivity, figure of merit, penetration depth and average field enhancement. As shown in Fig. 2(a), the RI sensitivity increases firstly with increasing the thickness of silver film and then keeps almost unchanged when the thickness is over $40 \mathrm{~nm}$. However, the conclusion is not applicable when the number of graphene is over 6 layers and the RI sensitivity would increase first and then decrease as the thickness of silver film increases. Thus, in order to obtain a greater RI sensitivity, the optimal thickness range of silver film is $40 \sim 80 \mathrm{~nm}$. With increasing graphene layers, the RI sensitivity also shows a significant upward trend at the optimal thickness of silver film, as shown in Fig. 2(a), and the highest RI sensitivity could reach approximately $140 \mathrm{deg} / \mathrm{RIU}$, while the RI sensitivity for monolayer silver SPR sensor is about $116 \mathrm{deg} / \mathrm{RIU}$. Other sensing properties, such as figure of merit, penetration depth and average field enhancement factor all have an optimal thickness range for silver film as shown in Fig. 2(b), 2(c) and 2(d), respectively. Different from RI sensitivity, they all showed a significant decreasing trend with the increase of graphene layers, which might be related to the increased angular peak width and decreased coupling efficiency at the resonance angle with the increase of graphene layers, as shown in Fig. 2(e), the reflectivity vs incident angle spectrum. That means, the increased number of graphene layers would widen the spectrum of reflectivity vs incident angle and decrease the intensity of transmitted beam. 

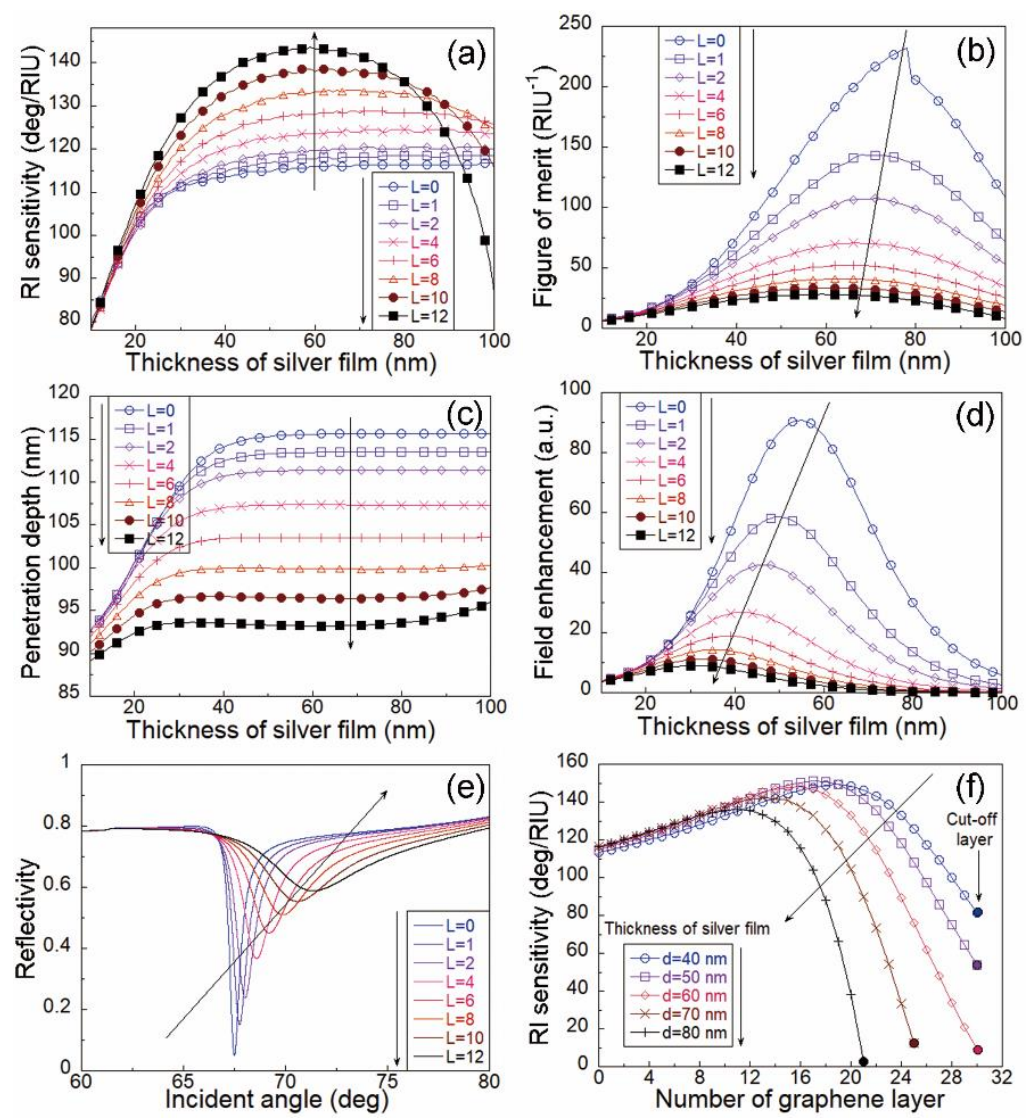

Fig. 2 The effect of different graphene layers and silver film thickness on the sensing performance without dielectric layer, (a) RI sensitivity, (b) figure of merit, (c) penetration depth away from sensor/sensing medium interface, (d) average field enhancement factor, (e) the changes of angular spectrum with 60 -nm silver film, and RI sensitivity (f) with larger change of graphene layers

Pay attention that, Fig. 2(e) also shows that the resonance angle has a red shift with increasing graphene layers, which might be the reason why the RI sensitivity was improved when adding the number of graphene as shown in Fig. 2(a). Further, we enlarged the variation range of graphene layer and calculated the corresponding RI sensitivity at different thicknesses of silver film as shown in Fig. 2(f). One can easily find that the RI sensitivity climbs up and then declines with respect to the number of graphene layer and there is a cut-off number of graphene layer. And the highest RI sensitivity is about $150 \mathrm{deg} / \mathrm{RIU}$ for graphene/silver/BK7 based SPR sensor with 40$\mathrm{nm}$ silver film and 20-layer graphene 
In order to clarify the influence of dielectric layer on the sensing performance, relevant theoretical calculation based on the multilayer reflection theory and the transmission matrix method was also carried out, as shown in Fig. 3. According to the above theoretical calculation results, the thickness of silver film and the number of graphene are set as $60 \mathrm{~nm}$ and 12 layers, respectively. And the optical parameters of the dielectric layer are thickness and refractive index, which are $0 \sim 30 \mathrm{~nm}$ and $1.35 \sim 5$ RIU, respectively, as shown in Table 1.
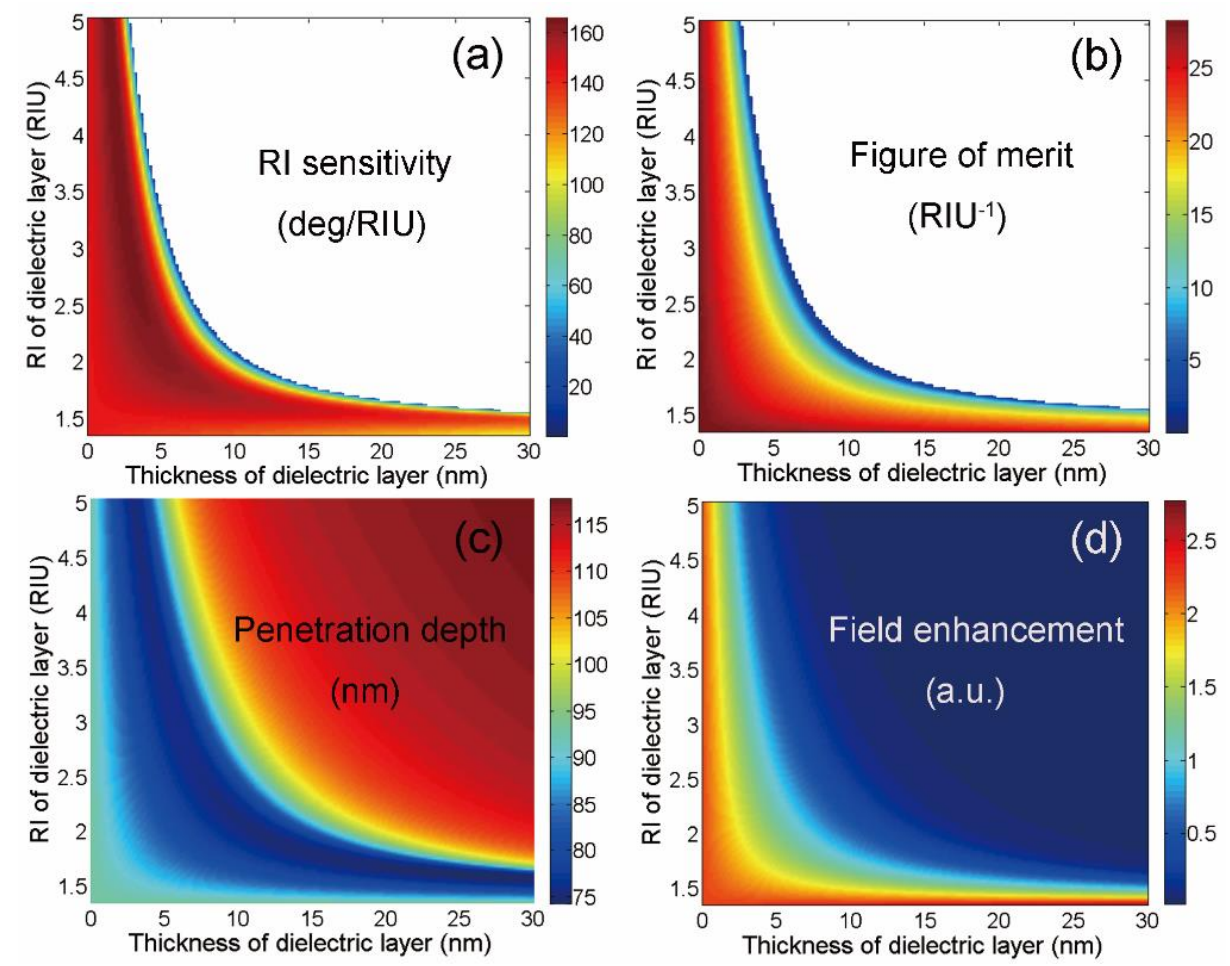

Fig. 3 The influence of the dielectric layer with different thickness and RI on the sensing performance, (a) RI sensitivity, (b) figure of merit, (c) penetration depth in sensing medium, (d) average field enhancement factor, with the 60-nm silver film and 12-layer graphene

For calculating the RI sensitivity, the RI difference of sensing medium is also set as $\triangle \mathrm{n}_{\mathrm{s}}=0.005$ RIU. Fig. 3 indicates that the RI sensitivity, figure of merit, penetration depth and average field enhancement factor almost all have a certain symmetrical relation with the thickness and RI of the dielectric layer. That means, the influences of the RI and thickness of dielectric layer on sensing properties are not isolated. The blank area in Fig. 3(a) and 3(b) means that SPR mode is nearly not generated at those 
situations or the difference of resonance angles is nearly zero and even negative when $\triangle \mathrm{n}_{\mathrm{s}}=0.005 \mathrm{RIU}$. Thus, those cut-off conditions are ignored in our later discussions. It can be obviously found that the RI sensitivity is positively correlated, while the other properties are negatively correlated with the RI or thickness of dielectric layer. The RI sensitivity is up to about $160 \mathrm{deg} / \mathrm{RIU}$ and is improved by $38 \%$ compared with that of monolayer silver film based SPR sensor. And the corresponding reduction rate of FoM and penetration depth are about $22 \%$ and $19 \%$, respectively. Therefore, optimization of dielectric layer structure can significantly improve the RI sensitivity of silver based SPR sensor.
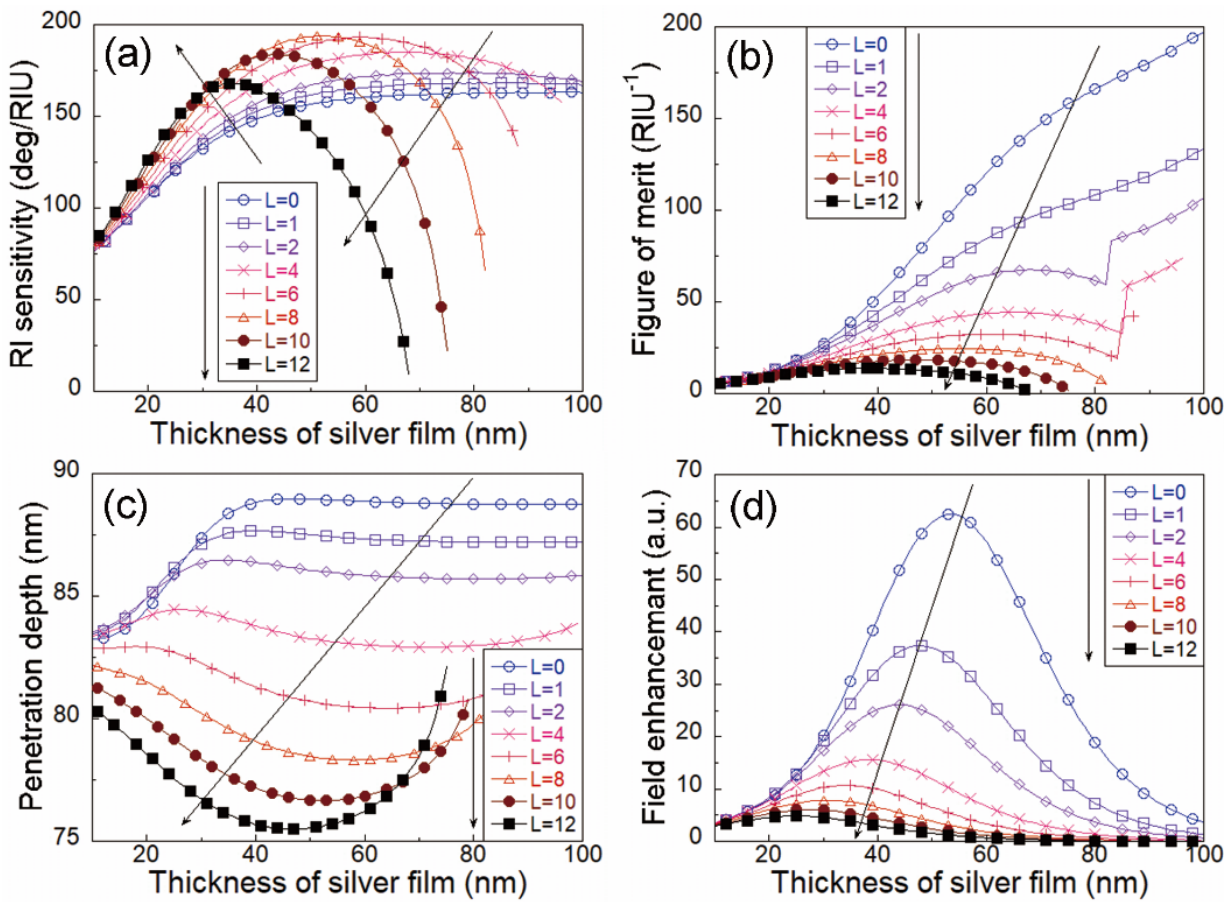

Fig. 4 The effect of different graphene layers and silver film thicknesses on the sensing performance, the dielectric layer thickness is $5 \mathrm{~nm}$ and the RI is 3 RIU, (a) RI sensitivity, (b) figure of merit, (c) penetration depth and (d) average field enhancement factor

As shown in Fig. 4, considering the presence of dielectric layer, for example, when the thickness is $5 \mathrm{~nm}$ and RI is $\mathrm{n}=3$, we also theoretically calculated the relationship between its sensing performance and the silver film thickness or the number of graphene layers. It can be obviously seen that there is also an optimal range for silver film thickness, that is $40 \sim 70 \mathrm{~nm}$, which is shorter than that without dielectric layer, 
that is $40 \sim 80 \mathrm{~nm}$. The largest RI sensitivity can reach nearly $200 \mathrm{deg} / \mathrm{RIU}$, which is $72 \%$ higher than that of the monolayer silver film based SPR sensor. Therefore, the graphene layer is more suitable as a protective or adsorbed layer and the optimized dielectric layer has a more obvious improvement on the RI sensitivity. Further, we also calculated RI sensitivity when the number of graphene layer is zero or monolayer via changing RI and thickness of dielectric layer, as shown in Fig. 5(a) and 5(b). The largest RI sensitivities for those cases are at least 450 and $350 \mathrm{deg} / \mathrm{RIU}$, respectively.
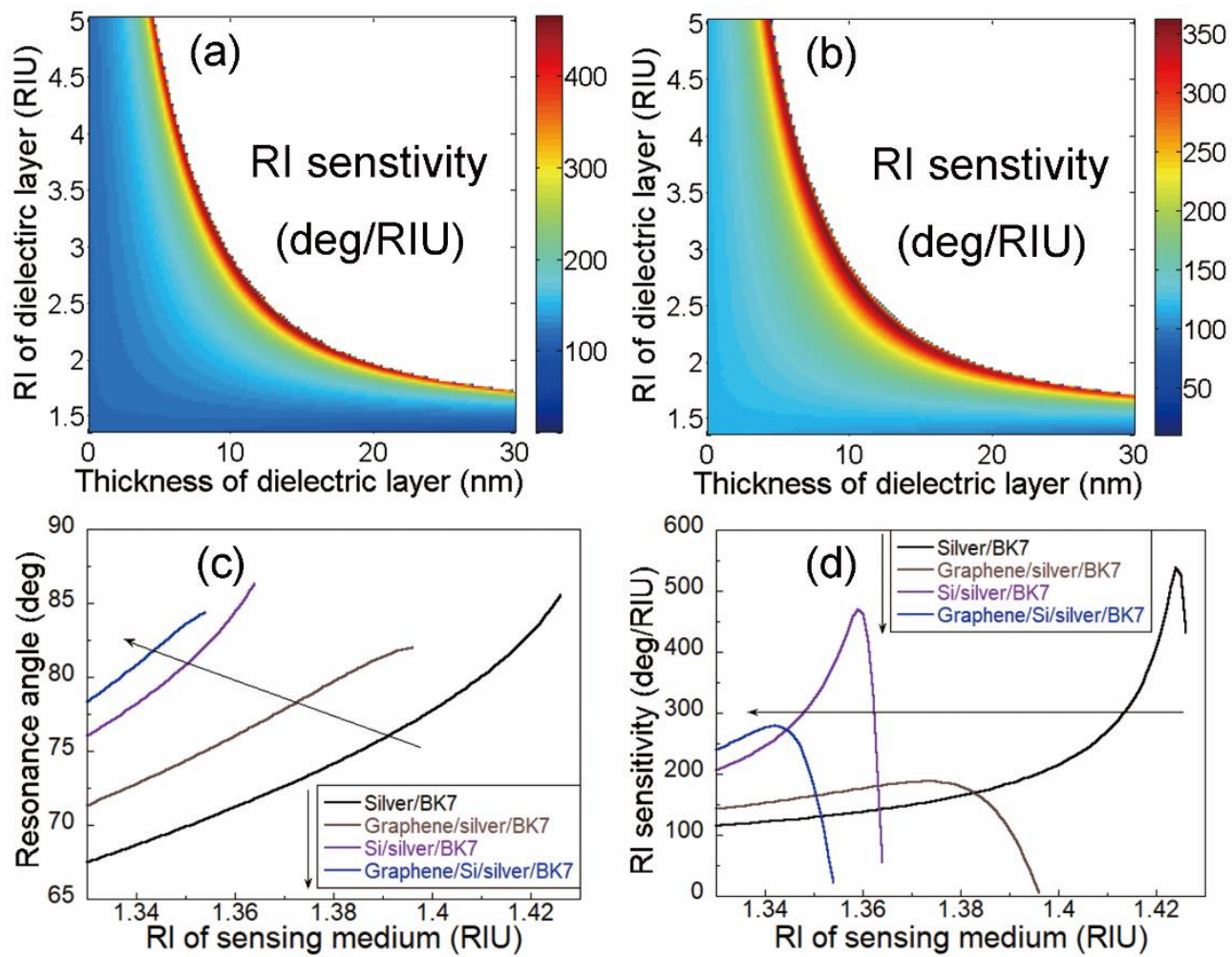

Fig. 5 Variation of RI sensitivity with respect to RI and thickness of dielectric layer for zerolayer graphene (a) and monolayer graphene (b), resonance angle (c) and RI sensitivity (d) depending on the RI of sensing medium for different sensing structures

The results obtained above could be summarized as that, both graphene and dielectric layer can enhance the RI sensitivity although the other evaluation indexes would be weakened and the former is more suitable as a protective or adsorbed layer and the latter has a more obvious improvement on the RI sensitivity. 


\section{Four Typical Silver Based SPR Sensor}

Based on the above discussions, four typical silver based SPR sensor would be discussed latter. The RI of different layers could be found from Table 1 and other calculating parameters of main layers are as shown in Table 2. It should be emphasized that Si material was selected as the dielectric layer and its imaginary part of RI was ignored.

Table 2 The parameters of different layers for calculating angular spectra

\begin{tabular}{ccc}
\hline Structures & Main layers & Parameters \\
\hline Silver/BK7 & Silver film & $55 \mathrm{~nm}$ \\
Graphene/silver/BK7 & Silver film & $60 \mathrm{~nm}$ \\
& Graphene & 12 layers \\
Dielectric layer & Silver film & $55 \mathrm{~nm}$ \\
(Si)/silver/BK7 & Dielectric layer (Si) & $3.846 \mathrm{RIU}, 5 \mathrm{~nm}$ \\
& Silver film & $55 \mathrm{~nm}$ \\
Graphene/Si/silver/BK7 & Dielectric layer (Si) & $3.846 \mathrm{RIU}, 5 \mathrm{~nm}$ \\
& Graphene & 3 layers \\
\hline
\end{tabular}

Fig. 5(c) and 5(d) show the resonance angle and RI sensitivity for the four typical sensing structures at different RI of sensing medium. The RI sensitivity is also calculated when RI difference of sensing medium is 0.005 RIU. The sensing range is also an important performance evaluation parameter for the application of SPR sensor. The whole sensing range combined with linear and nonlinear intervals can be ordered by silver/BK7 > graphene/silver/BK7 or $\mathrm{Si} /$ silver/BK7 $>$ graphene/Si/silver/BK7 as shown in Fig. 5(c). Fig. 5(d) also obviously indicates that the linear sensing range decreases when the silver film was protected with dielectric layer or graphene.

Fig. 6(a) shows that the RI sensitivity is $116 \mathrm{deg} / \mathrm{RIU}$ for conventional monolayer silver based SPR sensor in water environment. Fig. 6(b) demonstrates that the RI sensitivity would be improved $22.4 \%$ that is $142 \mathrm{deg} / \mathrm{RIU}$, when introducing a 12 -layer graphene on the silver film. It is demonstrated that the RI sensitivity could reach 206 
deg/RIU when the thickness of Si protected layer is $5 \mathrm{~nm}$, as shown in Fig.6(c). Further, as shown in Fig. 6(d), the RI sensitivity as high as $240 \mathrm{deg} / \mathrm{RIU}$ was obtained for the graphene/Si/silver/BK7 based SPR sensor.
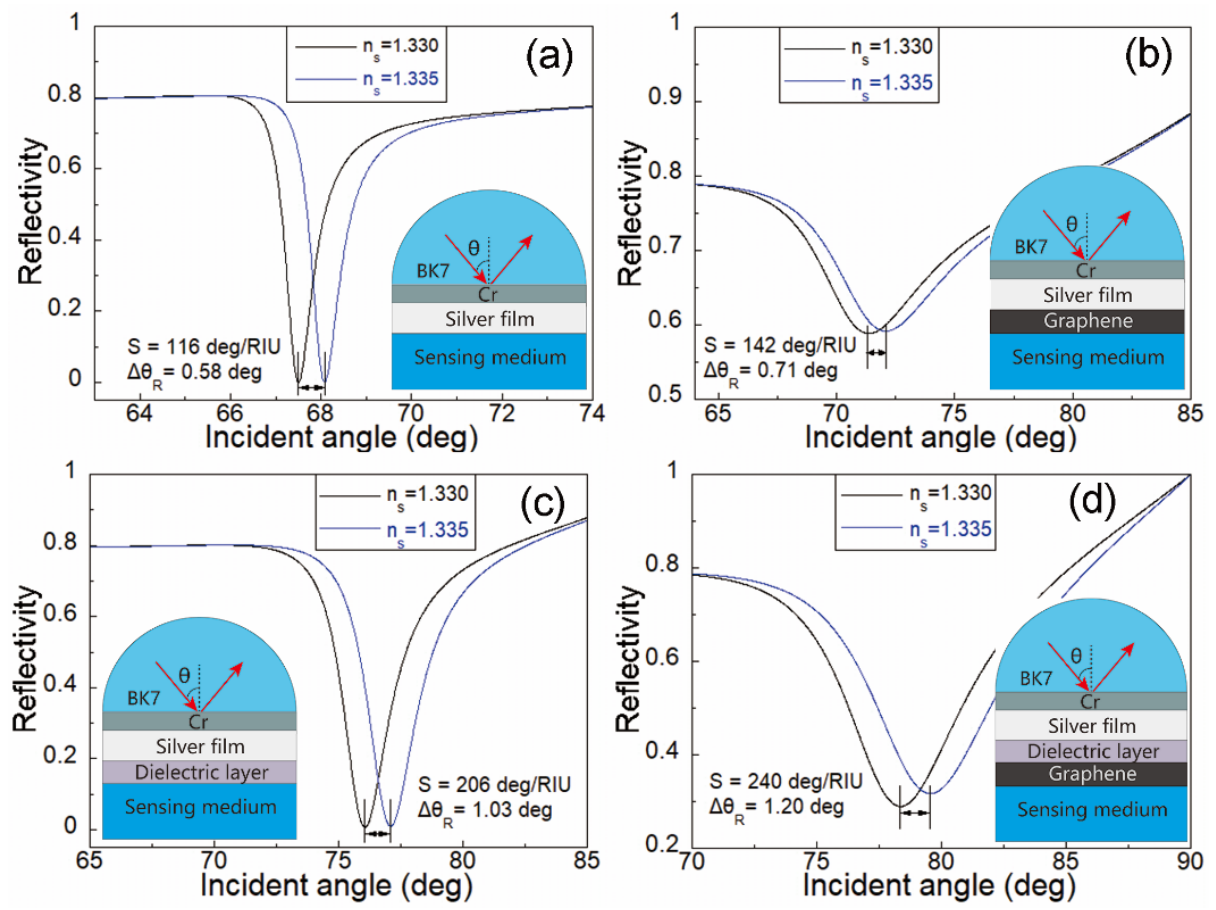

Fig. 6 Four typical silver based SPR sensing structures with their angular spectra and RI sensitivity simulated by Fresnel calculations. (a) Silver/BK7 structure, (b) graphene/silver/BK7 structure, (c) Si/silver/BK7 structure, (d) graphene/Si/silver/BK7 structure

Fig. 7 shows the electric filed distributions for four typical sensing structures. It is obviously that their electric field distributions are not continuous at every interface since the SPR phenomenon is excited under TM mode. The field intensity at $\mathrm{Cr} / \mathrm{BK} 7$ interface was used as a reference filed intensity for calculating field enhancement factor. As shown in Fig. 7, the electric filed intensity is nearly exponential attenuation in the sensing medium. It is also demonstrated that the existence of dielectric layer or graphene would significantly decrease the electric field intensity in the silver film and sensing medium. Especially, the graphene has a greater impact than the dielectric layer and it might be ascribed to its imaginary part of RI. 

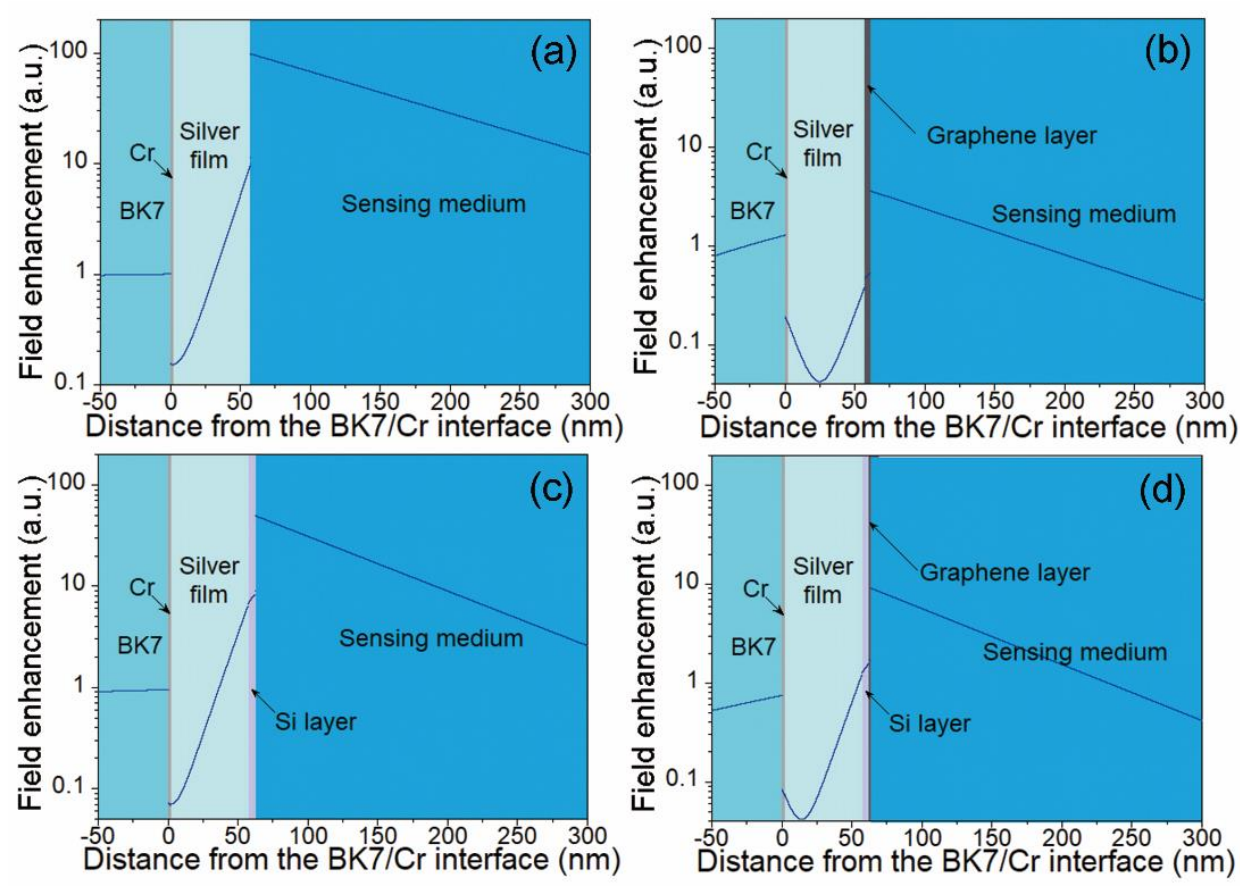

Fig. 7 Electric field enhancement distributions for the four typical silver based SPR sensing structures. (a) Silver/BK7 structure, (b) graphene/silver/BK7 structure, (c) Si/silver/BK7 structure, (d) graphene/Si/silver/BK7 structure

\section{Application of Biosensing with BSA Protein}

The biosensing application of optimized composite silver film based SPR sensor was carried out based on Fresnel reflection theory for BSA protein. Assuming adsorbed BSA molecules from sensing medium formed a monolayer on sensing surface as shown in insets of Fig. 8(a) and 8(b). Fig. 8(a) and 8(b) also show that the resonance angle is red shift with the increase of fBSA for both silver/BK7 and graphene/dielectric/silver/BK7 based SPR sensors. Fig. 8(c) presents shifts of the resonance angle vs fBSA for the typical four silver based SPR sensors. It is obviously that their detection sensitivities have an order of graphene/dielectric/silver/BK7 > dielectric/silver/BK7 > graphene /silver/BK7 $>$ silver/BK7. The shift of resonance angle for graphene/dielectric/silver/BK7 SPR sensor is 1.2, 2.1, and 3.1 folds larger than those of dielectric/silver/BK7, graphene /silver/BK7 and silver/BK7 SPR sensors, respectively. 

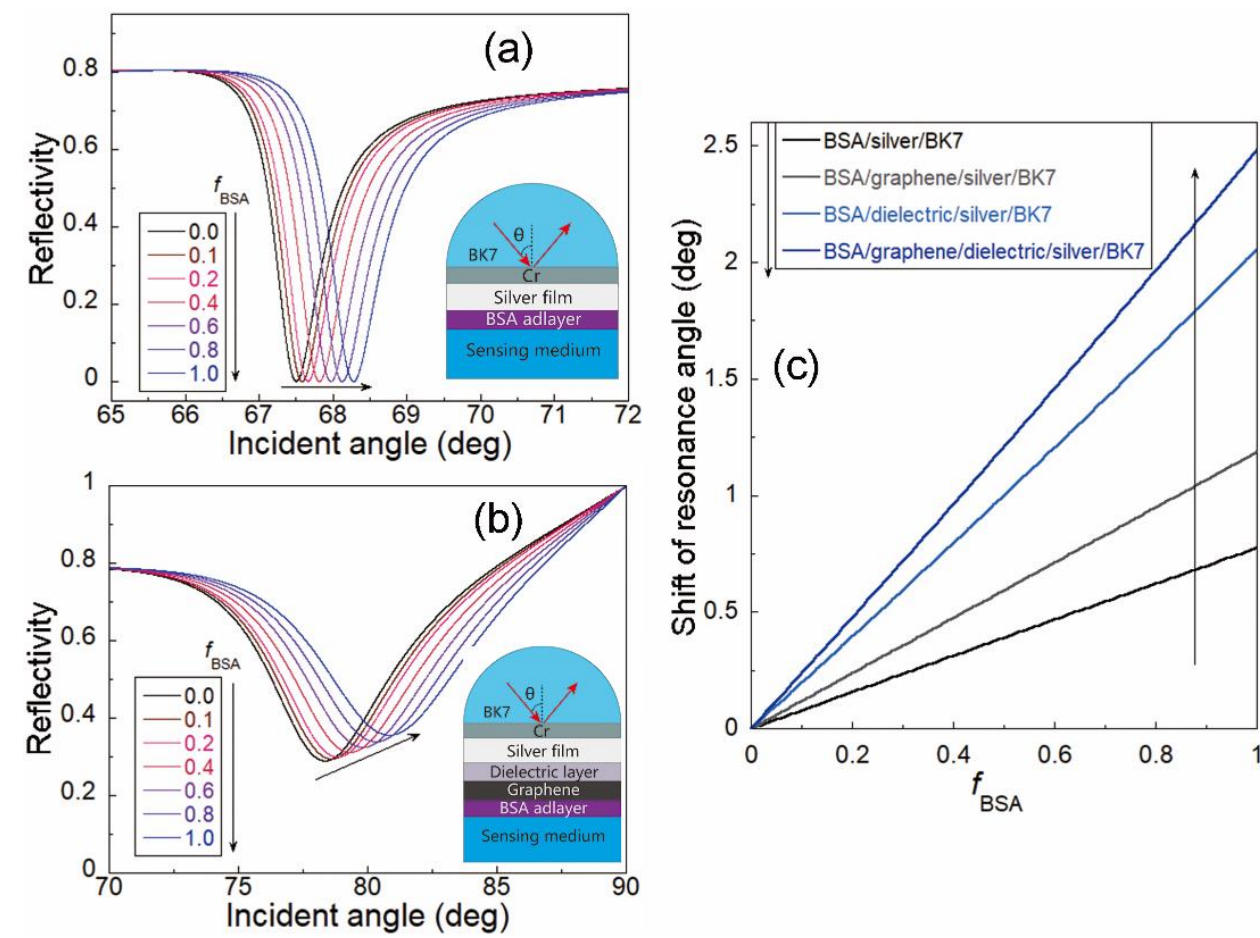

Fig. 8 Variation of reflection spectra with respect to the surface coverage of BSA molecules (fBSA) adsorbed on (a) the silver film (silver/BK7 structure) and (b) the graphene surface (graphene/dielectric/silver/BK7 structure), (c) shifts of the resonance angle vs fBSA for the typical four silver based SPR sensors

\section{Conclusion}

In this paper, we comprehensively discussed the influence of dielectric layer and graphene on silver based SPR sensor, the former factors are its refractive index and thickness and the latter is its number of layers. Graphene is a special two-dimensional nanomaterial, and thus the results are suitable for other two-dimensional nanomaterials, such as TMDCs and MXenes. The RI sensitivity could be obviously improved via introducing dielectric layer or graphene, while the other evaluation indexes, such as figure of merit, penetration depth, electric field enhancement and sensing range, are weakened. The RI sensitivity climbs up and then declines with respect to the number of graphene layer or RI and thickness of dielectric layer. The RI sensitivities for composite silver film based SPR sensor with monolayer silver, graphene/silver, dielectric/silver and graphene/dielectric/silver could reach about 116, 150, 450 and 350 
deg/RIU, respectively. Further, graphene is more suitable as a protective or adsorbed layer and the optimized dielectric layer has a more obvious improvement on the RI sensitivity. The shift of resonance angle for graphene/dielectric/silver/BK7 SPR sensor is 1.2, 2.1, and 3.1 folds than those of dielectric/silver/BK7, graphene /silver/BK7 and silver/BK7 SPR sensors, respectively.

Author Contribution G.W. contributed to this work in the modeling, calculations, and data analyzing. G.W. and L.H. contributed to this work in the data fitting. G.W. and L.H. prepared the paper. All authors read and approved the contents.

Funding Fundamental Research Funds for the Central Universities (JZ2020HGQA0143, JZ2021HGTA0142).

Data Availability The datasets used and/or analyzed during the current study are available from the corresponding author on reasonable request.

\section{Declarations}

Ethics Approval This is a theoretical study, and no ethical approval is required.

Consent to Participate Informed consent was obtained from all individual participants included in the study.

Consent for Publication The work described has not been published before. The work is not under consideration for publication elsewhere. Its publication has been approved by all co-authors.

Competing Interests The authors declare no competing interests.

\section{References}

1. Gao C, Lu Z, Liu Y, Zhang Q, Chi M, Cheng Q and Yin Y (2012) Highly Stable Silver Nanoplates for Surface Plasmon Resonance Biosensing. Angew Chem Int Ed 51(23):562933. 
2. Caucheteur C, Guo T and Albert J (2015) Review of plasmonic fiber optic biochemical sensors: improving the limit of detection. Anal Bioanal Chem 407(14):3883-97.

3. Wang ZY, Cheng ZQ, Singh V, Zheng Z, Wang YM, Li SP, Song LS and Zhu JS (2014) Stable and sensitive silver surface plasmon resonance imaging sensor using trilayered metallic structures. Anal Chem 86(3):1430-6.

4. Liu L, Wang M, Jiao L, Wu T, Xia F, Liu M, Kong W, Dong L and Yun M (2019) Sensitivity enhancement of a graphene-barium titanate-based surface plasmon resonance biosensor with an Ag-Au bimetallic structure in the visible region. J Opt Soc Am B 36(4):1108-16.

5. Pal S, Verma A, Prajapati YK and Saini JP (2020) Figure of Merit Enhancement of Surface Plasmon Resonance Biosensor Using Ga-Doped Zinc Oxide in Near Infrared Range. Photonic Sensors 10(4):340-52.

6. Jin Z, Guan WM, Liu C, Xue TY, Wang QY, Zheng WT and Cui XQ (2016) A stable and high resolution optical waveguide biosensor based on dense $\mathrm{TiO}_{2} / \mathrm{Ag}$ multilayer film. Appl Surf Sci 377(207-12.

7. Sun P, Wang M, Liu L, Jiao L, Du W, Xia F, Liu M, Kong W, Dong L and Yun M (2019) Sensitivity enhancement of surface plasmon resonance biosensor based on graphene and barium titanate layers. Appl Surf Sci 475(342-7.

8. Cheng ZQ, Wang ZY, Gillespie DE, Lausted C, Zheng Z, Yang M and Zhu JS (2015) Plain silver surface plasmon resonance for microarray application. Anal Chem 87(3):1466-9.

9. Wang G, Wang C, Yang R, Liu W and Sun S (2017) A sensitive and stable surface plasmon resonance sensor based on monolayer protected silver film. Sensors 17(12):2777.

10. Touahir L, Niedziolka-Joensson J, Galopin E, Boukherroub R, Gouget-Laemmel AC, Solomon I, Petukhov M, Chazalviel J-N, Ozanam F and Szunerits S (2010) Surface plasmon resonance on gold and silver films coated with thin layers of amorphous siliconcarbon alloys. Langmuir 26(8):6058-65.

11. Mudgal N, Saharia A, Choure KK, Agarwal A and Singh G (2020) Sensitivity enhancement with anti-reflection coating of silicon nitride $\left(\mathrm{Si}_{3} \mathrm{~N}_{4}\right)$ layer in silver-based Surface Plasmon Resonance (SPR) sensor for sensing of DNA hybridization. Appl Phys A: Mater Sci Process 126(12):946.

12. Tiwari K, Sharma SC and Hozhabri N (2016) Hafnium dioxide as a dielectric for highlysensitive waveguide-coupled surface plasmon resonance sensors. AIP Adv 6(4):045217.

13. Zhou J, Yang T, Chen J, Wang C, Zhang H and Shao Y (2020) Two-dimensional nanomaterial-based plasmonic sensing applications: Advances and challenges. Coord Chem Rev 410(213218.

14. Su S, Sun Q, Gu X, Xu Y, Shen J, Zhu D, Chao J, Fan C and Wang L (2019) Twodimensional nanomaterials for biosensing applications. TrAC, Trends Anal Chem $119(115610$

15. Zhao Z, Sun Y and Dong F (2015) Graphitic carbon nitride based nanocomposites: a review. Nanoscale 7(1):15-37.

16. Lin C and Chen S (2019) Design of high-performance Au-Ag-dielectric-graphene based surface plasmon resonance biosensors using genetic algorithm. J Appl Phys 125(11):113101.

17. Rouf HK and Haque T (2021) Sensitivity Enhancement of Graphene-MoSe $e_{2}$-Based SPR Sensor Using Ti Adhesion Layer for Detecting Biological Analytes. Plasmonics 10.

18. Wu L, Guo J, Wang Q, Lu S, Dai X, Xiang Y and Fan D (2017) Sensitivity enhancement by using few-layer black phosphorus-graphene/TMDCs heterostructure in surface plasmon resonance biochemical sensor. Sens Actuators B 249(542-8.

19. Xia G, Zhou C, Jin S, Huang C, Xing J and Liu Z (2019) Sensitivity enhancement of twodimensional materials based on genetic optimization in surface plasmon resonance. 
Sensors 19(5):1198.

20. Wu L, You Q, Shan Y, Gan S, Zhao Y, Dai X and Xiang Y (2018) Few-layer $\mathrm{Ti}_{3} \mathrm{C}_{2} \mathrm{~T}_{\mathrm{x}}$ MXene: A promising surface plasmon resonance biosensing material to enhance the sensitivity. Sens Actuators B 277(210-5.

21. Szunerits S, Maalouli N, Wijaya E, Vilcot J-P and Boukherroub R (2013) Recent advances in the development of graphene-based surface plasmon resonance (SPR) interfaces. Anal Bioanal Chem 405(5):1435-43.

22. Taniselass S, Arshad MKM and Gopinath SCB (2019) Graphene-based electrochemical biosensors for monitoring noncommunicable disease biomarkers. Biosens Bioelectron 130(276-92.

23. Anas NAA, Fen YW, Yusof NA, Omar NAS, Daniyal WMEMM and Ramdzan NS (2020) Highly sensitive surface plasmon resonance optical detection of ferric ion using CTAB/hydroxylated graphene quantum dots thin film. J Appl Phys 128(8):083105.

24. Vahed H and Nadri C (2019) Ultra-sensitive surface plasmon resonance biosensor based on $\mathrm{MoS}_{2}$-graphene hybrid nanostructure with silver metal layer. Opt Quantum Electron 51(1):20.

25. Vahed H and Nadri C (2019) Sensitivity enhancement of SPR optical biosensor based on Graphene- $\mathrm{MoS}_{2}$ structure with nanocomposite layer. Opt Mater 88(161-6.

26. Setareh M and Kaatuzian H (2021) Sensitivity enhancement of a surface plasmon resonance sensor using Blue Phosphorene/ $\mathrm{MoS}_{2}$ hetero-structure and barium titanate. Superlattices Microstruct 153(8.

27. Hansen WN (1968) Electric fields produced by the propagation of plane coherent electromagnetic radiation in a stratified medium. J Opt Soc Am 58(3):380-90.

28. Ding Y, Fan Y, Zhang YF, He YH, Sun SQ and Ma H (2015) Fabrication and optical sensing properties of mesoporous silica nanorod arrays. RSC Adv 5(110):90659-66.

29. Johnson PB and Christy RW (1974) Optical constants of transition metals: Ti, V, Cr, Mn, $\mathrm{Fe}, \mathrm{Co}, \mathrm{Ni}$ and Pd. Phys Rev B 9(12):5056-70.

30. Johnson PB and Christy RW (1972) Optical constants of the noble metals. Phys Rev B 6(12):4370-9.

31. Bruna M and Borini S (2009) Optical constants of graphene layers in the visible range. Appl Phys Lett 94(3):031901.

32. Ma K, Liu L, Zhang P, He Y and Peng Q (2019) Optimization of angle-pixel resolution for angular plasmonic biosensors. Sens Actuators B 283(188-97.

33. Wang G, Wang C and Sun S (2018) An optical waveguide sensor based on mesoporous silica films with a comparison to surface plasmon resonance sensors. Sens Actuators B 255(3400-8.

34. Hotta K, Yamaguchi A and Teramae N (2012) Nanoporous waveguide sensor with optimized nanoarchitectures for highly sensitive label-free biosensing. ACS Nano 6(2):1541-7.

35. Aspnes DE (1982) Optical properties of thin films. Thin Solid Films 89(3):249-62. 